\title{
Electrocoagulación y electroflotación para reducir contenido orgánico y turbidez en aguas residuales urbanas simuladas
}

\author{
Electrocoagulation and electroflotation to reduce organic \\ content and turbidity in simulated urban wastewater
}

\begin{abstract}
Ángel Villón', Warren Reategui'
${ }^{1}$ Facultad de Ingeniería Química y Textil, laboratorio 23 Universidad Nacional de Ingeniería (UNI), Av. Túpac Amaru 210, Rímac, Lima, Perú Recibido (Recieved): 05/09/2018 Aceptado (Accepted): 06/0/2019

\section{RESUMEN}

En un proceso de coagulación/floculación normal, el material coagulado/floculado se retira por sedimentación. La propuesta de la investigación es electrocoagularlos y flotarlos (electroflotación); para ello se produce $\mathrm{Al}^{+3}$ y Fe $\mathrm{Fe}^{+3}$ in-situ y oxígeno e hidrógeno, para hacer más eficiente este procedimiento (electroflotación), se usa un par de electrodos auxiliares, que su única función es producir estos gases $\mathrm{O}_{2} / \mathrm{H}_{2}$, de esta manera se retira los coágulos formados por la parte superior del sistema. La carga orgánica simulada proviene de la reducción del tamaño de especies como proteínas/carne, almidones/cereales, celulosa/verduras, grasas/aceites de origen vegetal y animal. El estudio logró reducir de 1200-1300 NTU de turbidez a valores por debajo de 16 NTU y de valores de 3,400 $\mathrm{mgL}^{-1}$ de DQO (Demanda química de oxígeno) a valores por debajo de $500 \mathrm{mgL}^{-1}$ de DQO.
\end{abstract}

Palabras clave: Electrocoagulación, electroflotación, DQO, aguas residuales

\begin{abstract}
In a normal coagulation / flocculation process, the coagulated / flocculated material is removed by sedimentation. The research proposal is to electrocoagulate and float them (electroflotation); For this, $\mathrm{Al}^{+3}$ and $\mathrm{Fe}^{+3}$ in-situ and oxygen and hydrogen are produced, in order to make this procedure more efficient (electroflotation), a pair of auxiliary electrodes is used, whose sole function is to produce these $\mathrm{O}_{2} / \mathrm{H}_{2}$ gases, This way the clots formed by the upper part of the system are removed. The simulated organic load comes from the reduction of the size of species such as proteins / meat, starches / cereals, cellulose / vegetables, fats / oils of vegetable and animal origin. The study was able to reduce turbidity of 12001300 NTU to values below 16 NTU and values of $3,400 \mathrm{mgL}^{-1}$ of COD to values below $500 \mathrm{mgL}^{-1}$ of COD.
\end{abstract}

Keywords: Electrocoagulation, electroflotation, COD, wastewater

\section{INTRODUCCIÓN}

La coagulación es una técnica muy utilizada en la reducción de turbidez y contenido orgánico DQO; demanda química de oxígeno, es una técnica química que permite establecer la presencia de compuestos de origen orgánico, usando reactivos apropiados como el $\mathrm{KMnO}_{4}$, dependiendo de la cantidad consumida se establecerá la cantidad de materia orgánica y se cuantifica como $\mathrm{mgL}^{-1 ;} \mathrm{y}$ esta consiste en agregar sales de $\mathrm{Fe}^{+3}$ (cloruro/ sulfato) y sales de $\mathrm{Al}^{+3}$ (sulfato), para que se formen los coágulos (uniones de coloides,

* Corresponding author:

E-mail: avillon@uni.edu.pe responsables de la turbidez y contenido orgánico), luego a través de movimiento mecánico (espesadores, decantadores) o con algún agente floculante, se permita que los coágulos, se separen y sean retirados luego, esto describe en forma muy breve el procedimiento de coagulación/floculación utilizado en forma estándar para el tratamiento de la turbidez y contenido orgánico. La propuesta de la investigación es la electrocoagulación, consiste en la generación insitu de estos iones de $\mathrm{Al}^{+3}$ y $\mathrm{Fe}^{+3}$, por la oxidación de ánodos de estos dos metales [1,2]. La propuesta de electroflotación, sustituiría a la decantación en un espesador (propuesta estándar), y esto implica el retiro de los coloides (coagulados) que provocan la turbidez y el DQO (carga orgánica), por la parte 
superior del recipiente, para que los coágulos pudieran flotar se utiliza la producción de $\mathrm{O}_{2}$ (ánodo) e $\mathrm{H}_{2}$ en el cátodo como gases para que provean el soporte de flotación necesario. Estos gases se producirían por el efecto de electrólisis del agua (el cual se produce si el potencial de trabajo supera el 1.1 $\mathrm{V}$, como es el caso) y la ineficiencia de los electrodos. [3]

La electrocoagulación es una propuesta que soluciona problemas de logística, reduce compra y almacenaje de reactivos químicos y se producirá cuando es necesario, la electroflotación evita las operaciones de decantación y espesamiento normalmente usado cuando existen precipitaciones o formación de sólidos, por lo tanto evita la compra de los equipos asociados a estas operaciones. Los residuos ocasionados por la electrocoagulación son retirados en forma de espuma sobre la celda y tratados posteriormente mediante una filtración.

\subsection{Mecanismos de coagulación}

El mecanismo de coagulación, tiene dos propuestas que explicarían este fenómeno, uno es reconocido como efecto eléctrico (electroestático o físico) y el otro conocido como efecto químico.

El primero se basa en la teoría de la doble capa, según esta teoría, la carga negativa de los coloides provocada por sus grupos funcionales carboxílicos, sulfatos, hidroxilos, fosfatos es desestabilizada por la carga positiva de los iones coagulantes. Por la propia naturaleza (carga negativa) de los coloides existe una fuerza de repulsión entre los coloides (lo que permite que siempre este en suspensión) por el efecto de su propia carga, el ion coagulante se acerca a la superficie del coloide, lo que hace que su potencial (potencial ?) se reduzca a tal punto que comienza a presentarse las fuerzas de atracción (tipo Van der Waals) entre los coloides y el ion coagulante comienzan a formar coágulos, de tal manera que el acercamiento de los iones coagulantes $\mathrm{Fe}^{+3}$ y $\mathrm{Al}^{+3}$, al coloide empujan la doble capa obligando a que su potencial se reduzca y posibilitando que haya una interacción ente los coloides provocando la formación de coágulos. Por lo tanto según esta teoría cuanto más iones coagulantes haya el efecto de coagulación sería más intenso, pero eso no es así, ocurre el efecto contrario y empieza a reducirse la presencia de coágulos y esto se explicaría porque cuanto más iones con carga positiva haya, estos comienzan a interactúa entre sí, y esto demostraría como se verá que cuanto más alta sea la concentración de iones coagulantes la turbidez no se reduce.

El efecto químico es el más sencillo en reconocerlo, se basa en la formación de pseudopolímeros del ión $\mathrm{Al}^{+3}$
[4], con los agentes causantes de la alcalinidad del agua y con el agua misma, como:

$$
\begin{aligned}
& \quad \mathrm{Al}\left(\mathrm{H}_{2} \mathrm{O}\right)_{6}^{+3}+\mathrm{OH}^{--} \rightarrow \mathrm{Al}\left(\mathrm{H}_{2} \mathrm{O}\right)_{5}(\mathrm{OH})^{+2}+\mathrm{H}_{2} \mathrm{O} \\
& \mathrm{Al}\left(\mathrm{H}_{2} \mathrm{O}\right)_{6}^{+3}+\mathrm{CO}_{3}^{-2} \rightarrow \mathrm{Al}\left(\mathrm{H}_{2} \mathrm{O}\right)_{5}(\mathrm{OH})^{+2}+\mathrm{HCO}_{3}^{-1} \\
& \mathrm{Al}\left(\mathrm{H}_{2} \mathrm{O}\right)_{6}^{+3}+\mathrm{HCO}_{3}^{-1} \rightarrow \mathrm{Al}\left(\mathrm{H}_{2} \mathrm{O}\right)_{5}(\mathrm{OH})^{+2}+\mathrm{H}_{2} \mathrm{CO}_{3} \\
& \text { Y con el agua: } \\
& \mathrm{Al}\left(\mathrm{H}_{2} \mathrm{O}\right)_{6}^{+3}+\mathrm{H}_{2} \mathrm{O} \rightarrow \mathrm{Al}\left(\mathrm{H}_{2} \mathrm{O}\right)_{5}(\mathrm{OH})^{+2}+\mathrm{H}_{3} \mathrm{O}^{+}
\end{aligned}
$$

La especie $\mathrm{Al}\left(\mathrm{H}_{2} \mathrm{O}\right)_{5}(\mathrm{OH})^{+2}$ es inestable y se forma $\mathrm{Al}_{2}\left(\mathrm{H}_{2} \mathrm{O}\right)_{8}(\mathrm{OH})_{2}^{+4}$ formándose un dimero, existiendo además otros como $\mathrm{Al}_{8}(\mathrm{OH})_{20}^{+4}, \mathrm{Al}_{7}(\mathrm{OH})_{17}^{+3}, \mathrm{Al}_{13}(\mathrm{OH})_{34}^{+5}, \mathrm{Al}_{6}(\mathrm{OH})_{15}^{+3}$.

Formación de pseudopolímeros del ión $\mathrm{Fe}^{+3}$, con los agentes causantes de la alcalinidad del agua y con el agua misma, como:

$\mathrm{Fe}\left(\mathrm{H}_{2} \mathrm{O}\right)_{6}^{+3}+\mathrm{OH}^{-} \rightarrow \mathrm{Fe}\left(\mathrm{H}_{2} \mathrm{O}\right)_{5}(\mathrm{OH})^{+2}+\mathrm{H}_{2} \mathrm{O}$

$\mathrm{Fe}\left(\mathrm{H}_{2} \mathrm{O}\right)_{6}^{+3}+\mathrm{CO}_{3}^{-2} \rightarrow \mathrm{Fe}\left(\mathrm{H}_{2} \mathrm{O}\right)_{5}(\mathrm{OH})^{+2}+\mathrm{HCO}_{3}^{-1}$

$\mathrm{Fe}\left(\mathrm{H}_{2} \mathrm{O}\right)_{6}^{+3}+\mathrm{HCO}_{3}^{-1} \rightarrow \mathrm{Fe}\left(\mathrm{H}_{2} \mathrm{O}\right)_{5}(\mathrm{OH})^{+2}+\mathrm{H}_{2} \mathrm{CO}_{3}$

$\mathrm{Y}$ con el agua:

$\mathrm{Fe}\left(\mathrm{H}_{2} \mathrm{O}\right)_{6}^{+3}+\mathrm{H}_{2} \mathrm{O} \rightarrow \mathrm{Fe}\left(\mathrm{H}_{2} \mathrm{O}\right)_{5}(\mathrm{OH})^{+2}+\mathrm{H}_{3} \mathrm{O}^{+}$

En el caso del hierro también forman dimeros como $\mathrm{Fe}_{2}\left(\mathrm{H}_{2} \mathrm{O}\right)_{8}(\mathrm{OH})_{2}^{+4}$.

Estas especies poliméricas son adsorbidas en la superficie del coloide desestabilizándolo, la otra forma es barriendo los coloides con estas formas pseudopoliméricas y de esta forma agrupándolas.

Ninguno de estos mecanismos el electroestático o el químico de por sí, logran explicar el fenómeno de la coagulación, por lo que ambos se complementan para entender este fenómeno, por ejemplo el mecanismo electroestático, no explicaría, cómo al haber más concentración de iones coagulantes, el efecto es inverso y disminuye el poder coagulante a mayor concentración, y en todo caso el mecanismo químico, dependería parte de su éxito del nivel de alcalinidad que tendría el agua. [5]

\subsection{Antecedentes}

La energía eléctrica fue propuesto para el tratamiento de agua por primera vez en Inglaterra en 1889 [6]. Y la primera que se aplicó la electrocoagulación para el tratamiento de agua potable a gran escala fue en Estados Unidos en 1946 [7]. Debido a que el costo de inversión de capital y el costo de la energía fue muy grande, no se reprodujo su uso en todo el mundo [8]. Sin embargo, debido a la extensa investigación en Estados Unidos y Rusia, durante los siguientes años el proceso ha ganado gran cantidad de conocimiento e información, más aún con la importancia que fue alcanzando en la normativa ambiental sobre las aguas residuales y con esto se han recuperado las tecnologías electroquímicas, para uso de tratamiento de aguas residuales, tan es así que su importancia ha 
sido privilegiado durante las últimas dos décadas. [9]. Hoy en día, las tecnologías electroquímicas han alcanzado un estado que no solo son comparables con otras tecnologías en términos de costo pero también potencialmente más eficientes y para algunas situaciones particulares las tecnologías electroquímicas son indispensable en el tratamiento aguas residuales que contienen contaminantes refractarios [10].

\subsection{Consideraciones previas experimentales}

Se ha podido verificar experimentalmente, que la oxidación del $\mathrm{Fe} \mathrm{a} \mathrm{Fe}^{+3}$ directamente desde el ánodo de hierro no es posible y esto se ha podido comprobar usando los indicadores colorimétricos, ferricianuro férrico y tiocianato de sodio, el primero identificador de $\mathrm{Fe}^{+2}$ y el segundo identificador de $\mathrm{Fe}^{+3}$, lo que si se ha comprobado es que el $\mathrm{Fe}$ pasa a $\mathrm{Fe}^{+2}$ electrolíticamente, por lo tanto se presume que el $\mathrm{Fe}^{+2}$ se oxida posteriormente a $\mathrm{Fe}^{+3}$ por la presencia de $\mathrm{O}_{2}$ en el medio, producto del oxígeno producido por la electrólisis del agua $2 \mathrm{H}_{2} \mathrm{O} \rightarrow 2 \mathrm{H}^{+}+\mathrm{O}_{2}+4 \mathrm{e}^{\text {. }}$.

En vista que era posible que la cantidad de gases que se produjeran en los electrodos (ánodo y cátodo) no fuera suficiente para producir la flotación esperada, el prototipo cuenta con dos electrodos (auxiliares) sumergidos en forma horizontal en el fondo del recipiente, el cual podría proveer de la cantidad de gas que fuera necesario para el objetivo figura 1.

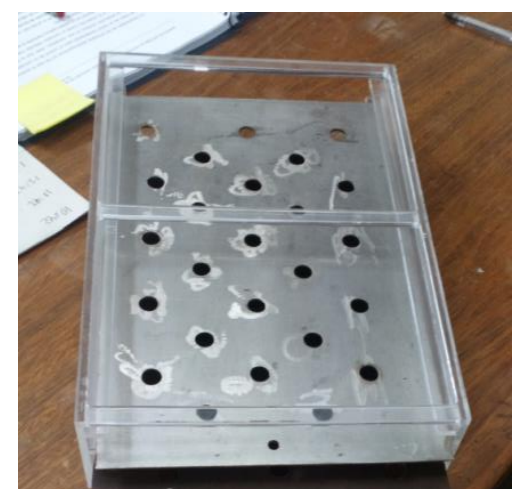

Figura 1. Electrodos auxiliares, ubicados en el fondo de la celda

El tamaño de la burbuja de gas influye en la capacidad de flotación de la burbuja, se demostrará en la parte experimental, que a menor velocidad de producción de estos gases, la burbuja será más pequeña y más uniforme en su desprendimiento y su distribución, colaborando mejor en el efecto de flotación, por lo tanto el tamaño de la burbuja será controlada por la cantidad de corriente utilizada en estos electrodos. La masa generada de iones coagulantes en el medio fue controlado con la cantidad de corriente utilizada en ellos, y el cálculo de la predicción de la cantidad se hizo utilizando la ley de Faraday, asumiendo un 100\% de eficiencia inicialmente, pero luego experimentalmente se halló su eficiencia, que estuvo cerca del $85 \%$, este valor de eficiencia real ayudo a corregir el tiempo que la corriente debería circular. Para la cantidad inicial en $\mathrm{g} \mathrm{L}^{-1}$ de cada ión (Fe y $\mathrm{Al}$ ) en el medio, para los fines deseados de reducir la turbidez y el DQO, se usó el dato bibliográfico [11] el cual recomienda 0.0792-0.1192 $\mathrm{g} \mathrm{Al}^{+3} \mathrm{~L}^{-1}$ y 0.2585-0.2985 g $\mathrm{Fe}^{+3} \mathrm{~L}^{-1}$, para una turbidez inicial de 1300-1750 NTU, estos valores iniciales fueron modificados en rangos hacia abajo y hacia arriba, para evaluar los efectos de estos cambios. Para estos fines la corriente como densidad de corriente juega un papel muy importante, porque define esas cantidades. Las eficiencias de los ánodos determino la cantidad de gramos de $\mathrm{Fe}^{+3}$ y $\mathrm{Al}^{+3}$ que realmente se generaron en el proceso electrolítico, para esto los ánodos de aluminio y hierro se pesaron antes y después de cada ensayo, y la diferencia de peso sirvió para evaluar la eficiencia utilizando la siguiente relación:

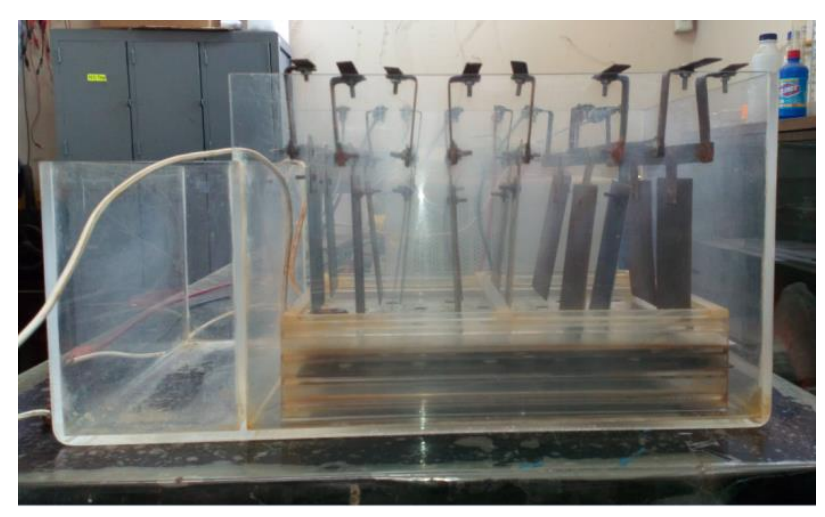

Figura 2. Disposición de los electrodos de trabajo y los cables blancos conectan a los electrodos que producen los gases.

\section{Eficiencia anódica

$$
=\frac{\text { Peso real pérdido }}{\text { Peso teórico perdido por la ley de Faraday }}
$$

Considerando que el peso teórico perdido, según la ley de Faraday se expresa que por cada 96,500 coulombs se genera en el ánodo 1 eq-g del elemento y en promedio fue de $85 \%$. 


\section{PARTE EXPERIMENTAL}

\subsection{Descripción del reactor electrolítico}

El sistema opera como un reactor electrolítico tipo batch a escala laboratorio, con capacidad para tratar 11 litros de aguas residuales (simulada), en la cual están sumergidos los electrodos (como ánodos), 4 electrodos de aluminio y 4 electrodos de acero comercial, dispuestos intercaladamente, con sus respectivos cátodos de acero comercial (como se comportan como cátodos no hay riesgo que se oxiden) estos electrodos son placas rectangulares metálicas de acero comercial $62.6 \mathrm{~cm}^{2}$ y aluminio 61.6 $\mathrm{cm}^{2}$, dispuestas en paralelo y conectadas a fuente de corriente continua que proporciona la energía eléctrica (corriente eléctrica) requerida para la electrocoagulación. Inicialmente se usó la misma corriente para los dos tipos de ánodos y luego se discriminará (separará) según las necesidades de masa de cada uno de ellos.

En la figura 2, además de los 4 electrodos de hierro y los cuatro de aluminio, con sus respectivos cátodos, se puede apreciar los electrodos generadores de gases únicamente, colocados en la parte inferior del recipiente, sobre un soporte de acrílico. Los electrodos están soportados sobre barras de cobre que sirven de conductores eléctricos. La fuente de corriente continua brinda $6 \mathrm{~A}$ a $32 \mathrm{~V}$, cuando hubo necesidad de más energía se contó con 3 de estos equipos, además posee controles de corriente y voltaje regulables e independientes (marca Siglent, SPD3303C)), la corriente se puede regular hasta en una centésima de amperio y es auto regulable (mantiene constante la corriente a pesar de haber fluctuaciones en el sistema).

Además se tomaron medidas del potencial eléctrico de los electrodos de hierro y aluminio, usando para ello un electrodo de referencia de $\mathrm{Ag} / \mathrm{ClAg}$ (marca Hanna) y un multímetro (marca Sanwa, modelo CD771), se tomaron medidas de $\mathrm{pH}$ y te temperatura.

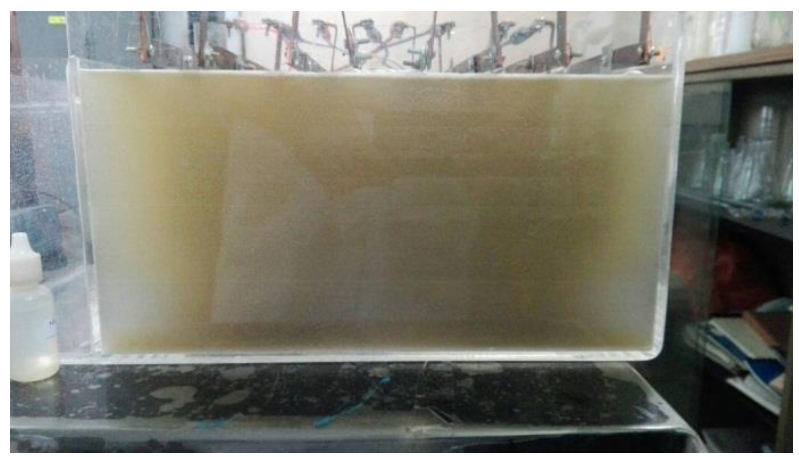

Figura 3. Apariencia del medio al inicio del proceso de electrocoagulación y electroflotación

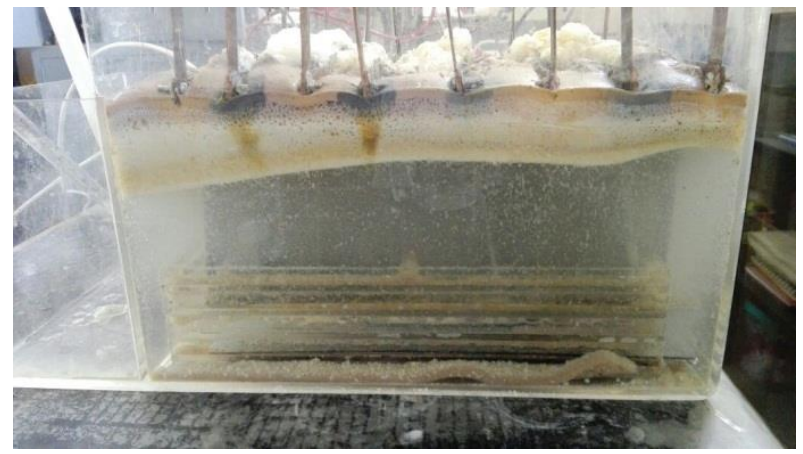

Figura 4. Apariencia del medio al final del proceso de electrocoagulación y electroflotación.

Los ensayos experimentales duraron la mayor parte 2:40 horas, de las cuales 1 hora fueron utilizadas para generar iones coagulantes y gases desde los electrodos inferiores y 1:40 horas adicionales de producción de gases (gases en total 2:40 horas). Debido al tiempo transcurrido de tener constantemente sometido el sistema al paso de una corriente sobre un medio líquido con una conductividad de $1.22 \mathrm{mScm}^{-1}$, esta producía temperaturas que oscilaba entre 40 a $50{ }^{\circ} \mathrm{C}$, la cual es la temperatura que se producía la electrocoagulación. La turbidez fue medida con un equipo marca Lovibond, modelo TB 210 IR, el DQO con un digestor (reactor), marca Lovibond, modelo RD 125 y un fotómetro marca Lovibond, modelo MD 200, las pruebas fueron hechas en viales (Lovibond) con una escala de o a 15,000 $\mathrm{mgL}^{-1}$ conteniendo permanganato de potasio y ácido sulfúrico.

\subsection{Regulaciones gubernamentales sobre calidad del agua final tratada.}

Los requerimientos de concentración de Fe dentro de las aguas residuales debe ser menor o igual a $7 \mathrm{mgL}^{-1} \mathrm{y}$ para el aluminio $10 \mathrm{mgL}^{-1}$, según los Estándares Nacionales de Calidad del Agua y el Protocolo de Monitoreo de Calidad del Agua-ANA (Autoridad Nacional del Agua), estas disposiciones limita el uso de Fe y Al para la coagulación [12,13]. La turbidez aceptada para descarga en las alcantarillas debe ser igual o menor de 20 NTU. Según las normas peruanas, el DQO final debe estar por debajo de $1000 \mathrm{mgL}^{-1}$ siempre y cuando esta agua será devuelta a sistemas de alcantarillado, ya que estas mismas normas ofrecen otro DQO final para cuando el agua sea devuelta a alguna fuente natural. [13,14].

Lo indicado en los párrafos anteriores determina parte de los objetivos experimentales definidos en la investigación. 


\subsection{Consideraciones y análisis experimentales}

El trabajo experimental consistió en mantener constantes dos parámetros, la turbidez inicial (constante) generada por la carga estandarizada orgánica simulada, lo que define un valor de turbidez y DQO iniciales aproximadamente similares, y un tiempo de proceso promedio de 2.5 horas. Las variables de operación fueron (1) las concentraciones de Fe y Al en el medio, (2) la velocidad de producción de esas concentraciones, (3) intensidad de corriente utilizada en lo electrodos auxiliares de producción de gases, (4) el tiempo de funcionamiento de los electrodos auxiliares. En cualquiera de los casos cuando una variable de operación se modificó, siempre se mantuvo constante las otras variables. Para verificar los resultados de cada ensayo o prueba, se utilizó la medición de turbidez y luego el DQO. La evaluación de éxito de la prueba se corroboró utilizando los valores máximos establecidos por la legislación nacional.

Los resultados obtenidos por los 28 ensayos son mostrados en la tabla 1, se usaron las corrientes que podían proveer la cantidad de Fe y Al en forma iónica. Hasta la prueba 9, se utilizó 150 minutos totales que incluía el uso de los electrodos auxiliares productora de los gases usando desde 2 a $12 \mathrm{~A}$, su uso es totalmente justificable debido a las altas eficiencias los ánodos, los cuales eran incapaces de producir los gases suficientes, para la electroflotación.

La turbidez final no estaba dentro del rango aceptado, excepto cuando se usó 2 A en los electrodos auxiliares. Esto se debe a que cuando menos intensidad de corriente se usa en estos electrodos las burbujas son más pequeña y más uniformes lo que logra una mejor flotabilidad a los coágulos formados.

Se hizo una prueba usando solamente aluminio (prueba 26) y sólo hierro (prueba 27), en el caso de sólo aluminio llego a las características deseadas 4.88 NTU y $578 \mathrm{mgL}^{-1}$ de DQO y con el hierro 21.2 NTU y 322 $\mathrm{mgL}^{-1}$ de DQO, este resultado indicó el uso en forma individual de los electrodos puede ser también eficiente, con la observación que el caso de Fe se usó $23 \%$ más de corriente eléctrica en relación a la usada en el Al. Es por esa razón que se redujo el tiempo de uso de los ánodos a 60 minutos desde la prueba 12 y la corriente utilizada 2.92 A según la recomendación de la bibliografía [11], pero los electrodos auxiliares se usaron 140 minutos. Al reducirse el tiempo de actividad de los ánodos y la corriente, lo que se busca es reducir la cantidad (masa) de los iones.
En relación a los electrodos auxiliares (inferiores) se puede notar en la tabla 1, que cuanto menor es la corriente eléctrica usada el resultado en turbidez es mejor y el mejor resultado es obtenido cuando se usó 2 A, Figuras 5 y 6.

Efecto: Tiempo Vs. DQO/Turbidez Al(3.22 A) $\mathrm{Fe}(3.48 \mathrm{~A})$ E.I.(2.0 A)

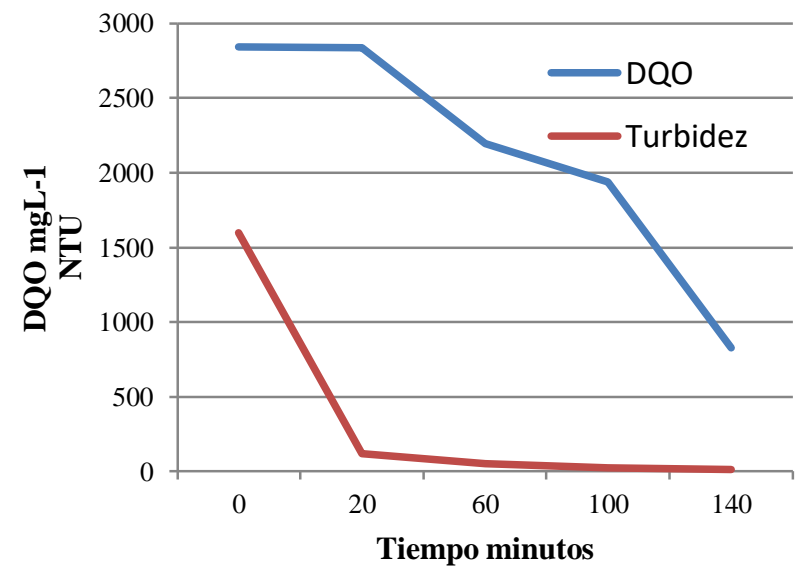

Figura 5. Evaluación del DQO/turbidez para corriente de $3.22 \mathrm{~A}$ en $\mathrm{Al}$ y $3.48 \mathrm{~A}$ para $\mathrm{Fe}$, y $2 \mathrm{~A}$ para los electrodos inferiores (EI), cortando la corriente de los electrodos a 140 minutos. Concentración residual de $\mathrm{Fe}=2.34 \mathrm{mgL}-1$ y Al= $8.56 \mathrm{mgL}-1$.

Efecto: Tiempo Vs. DQO/turbidez Al(1.94A) $\mathrm{Fe}(2.11 \mathrm{~A})$ E.I.(2.0A)

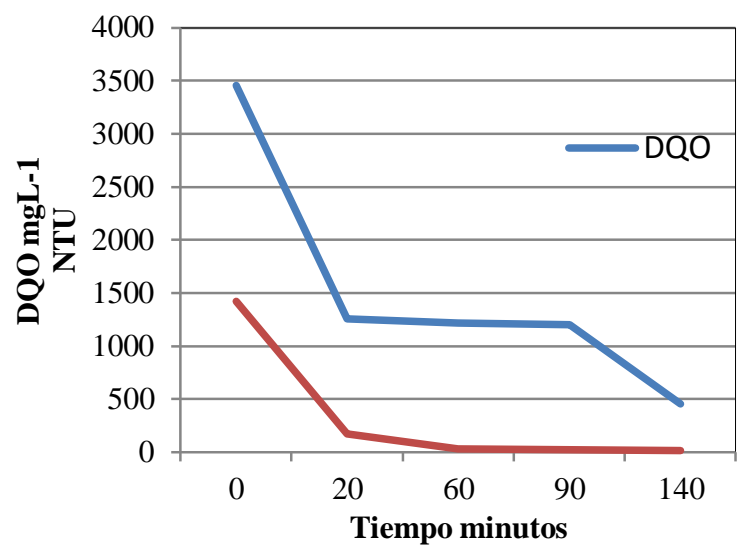

Figura 6. Evaluación del DQO/turbidez para corriente de 1.94 A en A y $2.11 \mathrm{~A}$ para $\mathrm{Fe}$, y $2 \mathrm{~A}$ para los electrodos inferiores, cortando la corriente de los electrodos a 140 minutos. Concentración final de $\mathrm{Fe}=3.19 \mathrm{mgL}-1$ y $\mathrm{Al}=10.71 \mathrm{mgL}-1$

De la prueba 12 a la 17 se usó la corriente que proveería la cantidad recomendada en la bibliografía 2.92 A [11] y se logró el objetivo holgadamente hasta llegar a 2 A en los electrodos auxiliares. 
En relación a la prueba 25 que corresponde al Figura 5 , se puede notar que con las condiciones de $3.22 \mathrm{~A}$ en $\mathrm{Al}$ y $3.48 \mathrm{~A}$ en $\mathrm{Fe}$ con $2 \mathrm{~A}$ en los electrodos auxiliares (electrodos inferiores), se logró una reducción del $70.8 \%$ del valor del DQO y $99.2 \%$ del valor de la turbidez.

En relación a la prueba 28 que corresponde al Figura 6, se puede notar que con las condiciones de 1.94 A en Al y $2.11 \mathrm{~A}$ en Fe con $2 \mathrm{~A}$ en los electrodos auxiliares (electrodos inferiores), se logró una reducción del $86.9 \%$ del valor del DQO y $98.7 \%$ del valor de la turbidez, con concentraciones residuales de Fe de 2.34 De la prueba 19 a la 23 se hizo lo mismo pero subiendo $110 \%, 120 \%, 130 \%$ sobre los valores base y los resultados no fueron mejores, se puede deducir que la abundancia de los iones entorpece la coagulación, desmereciendo la teoría electrostática que se supondría que cuanto más iones haya la coagulación sería mejor.

Las pruebas 24 a la 28 se midió el contenido de Al y Fe en las aguas resultantes de la electrocoagulación y electroflotación, mediante un ensayos de absorción atómica hecha en el laboratorio de la FIMM (UNI) y se observó que la prueba 25, muestra el contenido de turbidez y DQO en el agua tratada y el contenido de Al y $\mathrm{Fe}$ en el agua residual dentro los parámetros gubernamentales, aun partiendo con una de las turbideces más altas de 1600 NTU, y usando la más pequeña cantidad de $\mathrm{Fe}=3.24 \mathrm{~g}$ y $\mathrm{Al}=0.952 \mathrm{~g}$; aunque la prueba 28 se intentó agregar menos cantidad $\mathrm{Fe}=2.88 \mathrm{~g}$ y $\mathrm{Al}=0.876 \mathrm{~g}$, pero de una forma más lenta (en 90 minutos) y los resultados fueron muy aceptables en DQO y turbidez finales como de contenido de $\mathrm{Al} \mathrm{y} \mathrm{Fe}$ en las aguas tratadas, estos resultados fueron exitosos debido a que la evolución de los iones fueron reducidos y lentos.
$\mathrm{mgL}^{-1}$ y de Al $8.56 \mathrm{mgL}^{-1}$, y esto lo hace la mejor condición de operación para alcanzar el objetivo de reducir los valores de carga orgánica y turbidez a niveles por debajo del valor máximo admisible y además los valores de Fe y Al residual, por debajo de los valores máximos admisibles, estos resultados indican que la velocidad con la cual se producen los iones coagulantes, tienen que ser de tal manera que empate con la velocidad de formación de los coágulos, de otra manera una velocidad de producción mayor originaría un exceso no consumido y por lo tanto de baja eficiencia y mayor concentración en forma residual. 


\begin{tabular}{|c|c|c|c|c|c|c|c|c|c|c|c|c|c|c|}
\hline $\mathbf{P}$ & $\begin{array}{l}\text { Al } \\
\text { (A) }\end{array}$ & $\begin{array}{l}\mathrm{Fe} \\
\text { (A) }\end{array}$ & $\begin{array}{c}\mathrm{T} . \\
\text { total } \\
(\mathrm{min})\end{array}$ & $\begin{array}{c}\mathrm{T} . \\
\text { ánod. } \\
\text { (min) }\end{array}$ & $\begin{array}{c}\text { T. } \\
\text { aux. } \\
\text { (min) }\end{array}$ & $\begin{array}{c}\text { Ánodos } \\
\text { aux. } \\
\text { (A) }\end{array}$ & $\begin{array}{c}\text { Efi. Fe } \\
(\%)\end{array}$ & $\begin{array}{c}\text { Efi. Al } \\
(\%)\end{array}$ & $\begin{array}{l}\mathrm{Fe} \\
(\mathrm{g})\end{array}$ & $\begin{array}{l}\text { Al } \\
\text { (g) }\end{array}$ & $\begin{array}{c}\text { Turbidez } \\
\text { NTU } \\
\text { inicial }\end{array}$ & $\begin{array}{c}\text { Turbi- } \\
\text { dez } \\
\text { NTU } \\
\text { final }\end{array}$ & $\begin{array}{c}\text { DQO } \\
\text { Final } \\
\left(\mathrm{mgL}^{-1}\right)\end{array}$ & $\begin{array}{l}\text { Fe y Al } \\
\mathrm{mgL}^{-1} \\
\text { final }\end{array}$ \\
\hline 1 & 5.5 & 6.0 & 150 & 150 & - & - & 81.60 & 81.42 & 12.78 & 3.768 & 840 & 43.4 & - & \\
\hline 2 & 5.5 & 6.0 & 150 & 150 & 150 & 2.0 & 81.53 & 78.9 & 12.78 & 3.643 & 865 & 12.1 & - & \\
\hline 3 & 5.5 & 6.0 & 150 & 150 & 150 & 3.0 & 81.60 & 78.63 & 12.78 & 3.629 & 867 & 14.2 & - & \\
\hline 4 & 5.5 & 6.0 & 150 & 150 & 150 & 4.0 & 79.60 & 81.22 & 12.47 & 3.75 & 870.2 & 17.2 & - & \\
\hline 5 & 5.5 & 6.0 & 150 & 150 & 150 & 5.0 & 81.62 & 80.53 & 12.78 & 3.718 & 867.4 & 21.1 & - & \\
\hline 6 & 5.5 & 6.0 & 150 & 150 & 150 & 6.0 & 80.30 & 79.84 & 12.58 & 3.686 & 869.8 & 28.2 & - & \\
\hline 7 & 5.5 & 6.0 & 150 & 150 & 150 & 8.0 & 79.60 & 80.31 & 12.47 & 3.708 & 1113.2 & 30.5 & - & \\
\hline 8 & 5.5 & 6.0 & 150 & 150 & 150 & 10.0 & 80.57 & 79.82 & 12.62 & 3.685 & 996 & 32.8 & - & \\
\hline 9 & 5.5 & 6.0 & 150 & 150 & 150 & 12.0 & 80.76 & 78.91 & 12.65 & 3.643 & 985 & 39.4 & - & \\
\hline 10 & 5.5 & - & 150 & 60 & 150 & 2.0 & - & 85.45 & - & 1.607 & 1092 & 4.2 & - & \\
\hline 11 & - & 5.5 & 150 & 60 & 150 & 2.0 & 86.49 & - & 5.08 & - & 986 & 38.4 & - & \\
\hline 12 & 2.92 & 3.16 & 140 & 60 & 140 & 12.0 & 86.11 & 85.14 & 2.84 & 0.834 & 1350 & 23.1 & - & \\
\hline 13 & 2.92 & 3.16 & 140 & 60 & 140 & 10.0 & 88.24 & 85.10 & 2.91 & 0.833 & 1358 & 21.9 & - & \\
\hline 14 & 2.92 & 3.16 & 140 & 60 & 140 & 8.0 & 88.41 & 86.22 & 2.91 & 0.845 & 1354 & 19.9 & - & \\
\hline 15 & 2.92 & 3.16 & 140 & 60 & 140 & 6.0 & 88.27 & 86.14 & 2.91 & 0.844 & 1360 & 17.9 & - & \\
\hline 16 & 2.92 & 3.16 & 140 & 60 & 140 & 4.0 & 88.26 & 86.13 & 2.91 & 0.844 & 1380 & 16.1 & - & \\
\hline 17 & 2.92 & 3.16 & 140 & 60 & 140 & 2.0 & 88.24 & 86.12 & 2.91 & 0.846 & 1428 & 12.9 & - & \\
\hline 18 & 2.628 & 2.844 & 140 & 60 & 140 & 2.0 & 87.67 & 88.34 & 2.60 & 0.781 & 1450 & 17.1 & - & \\
\hline 19 & 2.336 & 2.528 & 140 & 60 & 140 & 2.0 & 88.14 & 83.35 & 2.32 & 0.705 & 1390 & 9.78 & - & \\
\hline 20 & 2.044 & 2.212 & 140 & 60 & 140 & 2.0 & 85.58 & 85.29 & 1.97 & 0.583 & 1410 & 18.8 & - & \\
\hline 21 & 3.212 & 3.476 & 140 & 60 & 140 & 2.0 & 89.31 & 89.31 & 3.24 & 0.972 & 1600 & 15.9 & - & \\
\hline 22 & 3.5 & 3.8 & 140 & 60 & 140 & 2.0 & 82.50 & 86.86 & 3.27 & 1.024 & 1340 & 15.8 & - & \\
\hline 23 & 3.8 & 4.11 & 140 & 60 & 140 & 2.0 & 89.16 & 87.42 & 3.82 & 1.151 & 1350 & 15.4 & - & \\
\hline 24 & 2.92 & 3.16 & 140 & 60 & 140 & 2.0 & 88.31 & 86.25 & 2.91 & 0.847 & 1650 & 12.9 & 871 & $\begin{array}{l}\mathrm{Fe}=6.09 \\
\mathrm{Al}=15.02\end{array}$ \\
\hline 25 & 3.212 & 3.476 & 140 & 60 & 140 & 2.0 & 89.31 & 88.25 & 3.24 & 0.952 & 1600 & 11.9 & 828 & $\begin{array}{l}\mathrm{Fe}=2.34 \\
\mathrm{Al}=8.56\end{array}$ \\
\hline 26 & 4.82 & - & 140 & 60 & 140 & 2.0 & - & 90.32 & - & 1.462 & 1780 & 4.88 & 598 & $\mathrm{Al}=10.99$ \\
\hline 27 & - & 5.96 & 140 & 60 & 140 & 2.0 & 89.45 & - & 5.17 & - & 1482 & 21.2 & 322 & $\mathrm{Fe}=5.93$ \\
\hline 28 & 1.94 & 2.11 & 140 & 90 & 140 & 2.0 & 87.14 & 89.48 & 2.88 & 0.876 & 1420 & 17.1 & 452 & $\begin{array}{l}\mathrm{Fe}=3.19 \\
\mathrm{Al}=10.71\end{array}$ \\
\hline
\end{tabular}

\section{CONCLUSIONES}

Existe una relación cinética entre la velocidad de producción de los iones y la velocidad con la que interactúan con los coloides (coagulación), el éxito de la coagulación dependerá en encontrar esa relación adecuada, y esto significa una producción lenta de iones.

La cantidad de masa de iones coagulantes producidos debe ser tal que cubra la necesidad de la coagulación y no produzca exceso que puede quedar presente en las aguas tratadas (iones residuales), esto se logró con $3.24 \mathrm{~g}$ de Fe y $0.952 \mathrm{~g}$ de Al (prueba 25), alcanzando al final un residual de $2.34 \mathrm{mgL}^{-1}$ de Fe y $8.56 \mathrm{mgL}^{-1} \mathrm{de} \mathrm{Al}$, los cuales están dentro de los VMA (valores máximos admisibles)
La prueba 28 demuestra (lo afirmado arriba), que al utilizar una corriente de $1.94 \mathrm{~A}$ para el Fe y $2.11 \mathrm{~A}$ para el Al, por 90 minutos, 30 minutos más sobre el estándar de las pruebas, eso significa menos corriente con un tiempo más prolongado (producción lenta de iones), originó el otro caso exitoso, en términos de DQO final $452 \mathrm{mgL}^{-1}$ reducción del $86.9 \%$ y de $98.7 \%$ en turbidez, y un contenido residual de Fe $3.19 \mathrm{mgL}^{-1} \mathrm{y}$ de Al de $10.71 \mathrm{mgL}^{-1}$.

El éxito de la electroflotación consiste en producir burbujas pequeñas (esto se logró con 2 A en los electrodos auxiliares $O$ inferiores) y seguir produciendo estos gases (en promedio 80 minutos más), luego de haber alcanzado la masa de iones de Fe y Al en el medio.

La producción de gases, $\mathrm{O}_{2}$ e $\mathrm{H}_{2}$ en el ánodo y cátodo es insuficiente para producir la electroflotación y se hace necesario electrodos auxiliares. 


\section{REFERENCIAS}

[1] M Yousuf A Mollah, Robert Schennach, Jose R Parga, David L Cocke 2001 Electrocoagulation (EC) - science and applications, Journal of Hazardous Materials 84 pp 29-41

[2] Morales B 2010 "Sistema de electrocoagulación como tratamiento de aguas residuales galvánicas" Ciencia e Ingeniería Neogranadina 20 pp 33-44

[3] George Z.KyzasKostas A.Mati 2016 Electroflotation process: A review, Journal of Molecular Liquids 220 pp 657-664

[4] Cogollo J 2011 "Clarificación de aguas usando coagulantes polimerizados; caso del hidroxicloruro de aluminio", DYNA 78 Número 165 pp 18-27

[5] Hector A.Moreno-Casillas David L.Cocke PaulMorkovsky J.R.Parga, Electrocoagulation

[6] M.Y.A. Mollah, R. Schennach, J.R. Parga and D.L. Cocke "Electrocoagulation (EC)-science and application," Journal of Hazardous Materials 84 pp. 29-41, 2001.

[7] E.E. Stuart 1946 Electronic water purification progress report on electronic coagulator. A new device which gives promise of unusually speedy and effective results, Water sewage, 84 pp 24-26

[8] Mehmet Kobya, Orhan Taner Can and Mahmut Bayramoglu 2003 "Treatment of textile wastewaters by electrocoagulation using iron and aluminum electrodes," Journal of Hazardous Material 100 pp 163-178

[9] G Chen, x Chen, P L Yue 2000 "Electrocoagulation and electroflotation of restaurant wastewater," J. Environ. Eng 126 pp. 858- 863

[10] GuohuaChen 2004 Electrochemical technologies in wastewater treatment Separation and Purification Technology 38, Issue 1, 15 pp 11-41

[11] Martinez N 2007“Tratamiento de Aguas Residuales Industriales mediante Electrocoagulación y Coagulación Convencional”. Tesis Ingeniería Química, Universidad De Castilla La Mancha

[12] Decreto Supremo Nºo2-2008-MINAM, "Estándares Nacionales de Calidad Ambiental del Agua". Normas Legales 377222. Edición El Peruano.

http://www.minam.gob.pe/calidadambiental/wpcontent/uploads/sites/22/2013/10/ds_002_2008_eca_agua.pd $f$

[13] Sedapal Lima-Perú "Valores Máximos Admisibles de las descargas de aguas residuales No domésticas". http://www.sedapal.com.pe/documents/10154/fedf8405-1bc2428e-9d8d-a1c2adoogf53

[14] Aguilar A 2015 “Evaluación de la eficiencia de una celda de electrocoagulación a escala laboratorio para el tratamiento de agua". Tesis Ingeniería Química, Universidad Mayor de San Marcos http://cybertesis.unmsm.edu.pe/bitstream/handle/cybertesis /4303/Aguilar_ae.pdf;jsessionid=000C98700BC63144704209 7352E8AF89? sequence $=3$

Los artículos publicados por TECNIA pueden ser compartidos a través de la licencia Creative Commons: CC BY-NCND 2.5 Perú. Permisos lejos de este alcance pueden ser consultados a través del correo revistas@uni.edu.pe 